\title{
A home for integrative structural models
}

\author{
PDB-Dev hosts integrative structural models of biomolecular assemblies solved by hybrid methods.
}

$\mathrm{T}$ he Protein Data Bank, or PDB, is an essential resource for archiving, validating and disseminating biomolecular structure models to a broad community. Currently over 135,000 structure models-determined by X-ray crystallography, nuclear magnetic resonance (NMR) spectroscopy and cryo-electron microscopy (cryo-EM) - can be found in the PDB.

Despite recent remarkable technological advances for structure analysis, especially in the field of cryo-EM, there are many large, complex, dynamic biomolecular assemblies that just cannot be solved with a single structure-determination technique. To solve these challenging structures, researchers can apply 'hybrid' methods-including lower-resolution approaches such as electron tomography, small-angle X-ray scattering, cross-linking-mass spectrometry and others-to generate spatial restraints. These spatial restraints provide key clues about the arrangement of assembly subunits and are applied in 'integrative' modeling pipelines for structure determination.

The PDB, however, does not have the infrastructure to handle models produced via hybrid methods. With the development of a prototype system, PDB-Dev, integrative models now have a home.

Integrative models pose several challenges for archiving. Such models may contain multiple resolution scales or conformational states, or may be represented as ensembles of structures. PDB-Dev was built on the existing data standard for the PDB, ensuring interoperability and extensibility. Besides the integrative models themselves, PDB-Dev archives the experimental spatial restraints, modeling pipelines used and associated metadata.
At the moment, preliminary validation metrics are available in PDB-Dev, but further community input will be needed to develop such metrics for integrative models.

PDB-Dev currently contains nine integrative models and is open for new submissions at https://pdb-dev.wwpdb. org. The resource will surely have a positive impact and facilitate greater reproducibility and transparency in structural studies of biological assemblies.

\section{Allison Doerr}

Published online: 31 May 2018

https://doi.org/10.1038/s41592-018-0032-0

Research papers

Vallat, B. et al. Development of a prototype system for archiving integrative/hybrid structure models of biological macromolecules. Structure https://doi. org/10.1016/j.str.2018.03.011 (2018).

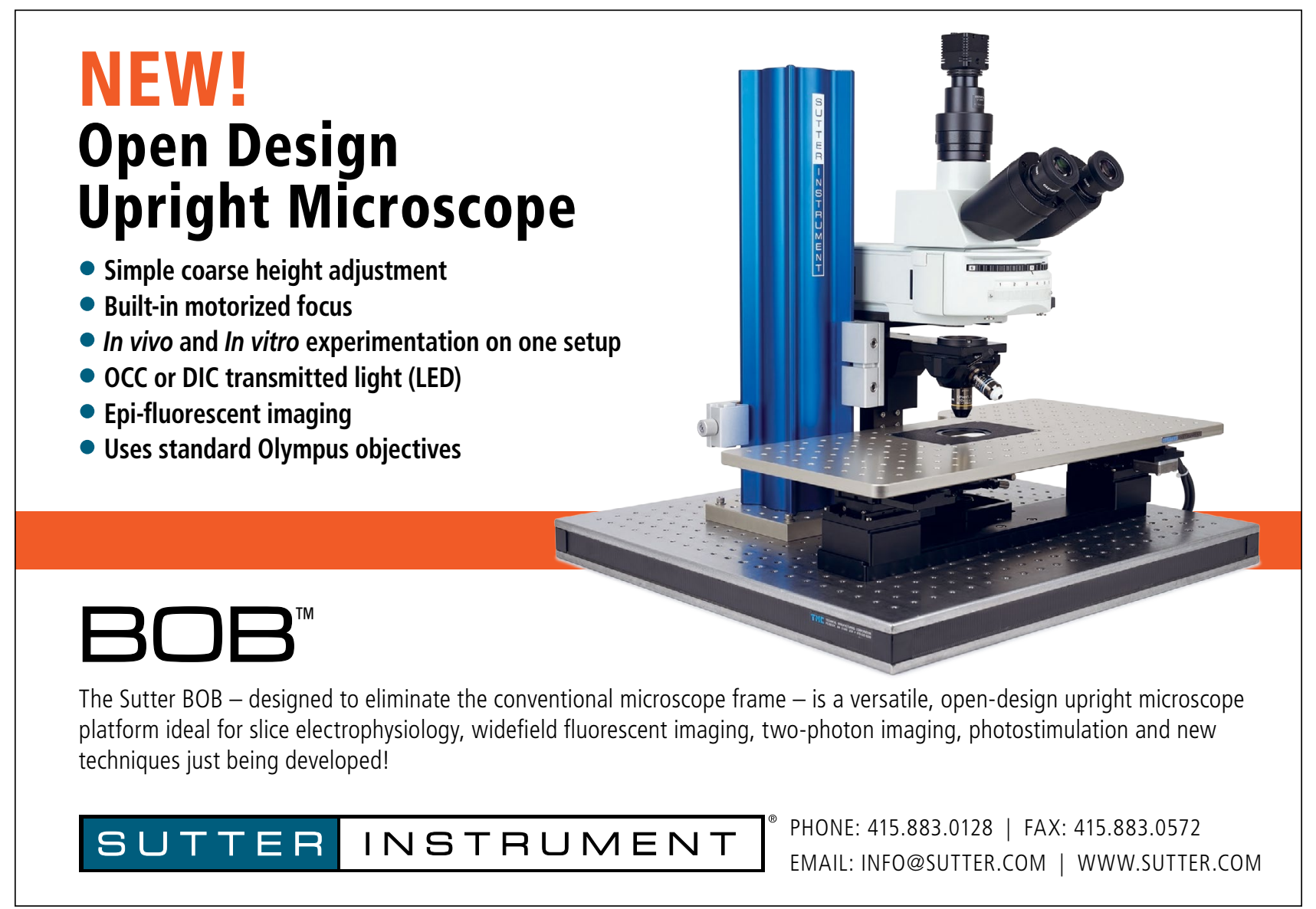

